\title{
GENETIC CONTROL OF RECOMBINATION IN SCHIZOPHYLLUM COMMUNE: SPECIFIC AND INDEPENDENT REGULATION OF ADJACENT AND NON-ADJACENT CHROMOSOMAL REGIONS
}

\author{
G. SIMCHEN* and JUDITH STAMBERG* \\ Department of Genetics, University of Birmingham, England; \\ Biological Laboratories, Harvard University, Cambridge, Mass., U.S.A.
}

Received 1.x.68

\section{INTRODUGTION}

Meiotic recombination is controlled by a series of genes. Unfortunately, only little is known about these genes and their actual role in the process of recombination. We have recently suggested that two types of gene systems may be involved in the control and regulation of recombination, the "coarse" and the "fine" (Stamberg, 1968; Simchen and Stamberg, 1969). These two types of control differ with regard to the following criteria: magnitude of effect on recombination, extent of genome affected, occurrence of natural variation, type of organism exhibiting the effect, and evolutionary role. The existence of " coarse " controls can be deduced from the complete failure of certain mutant genotypes to recombine altogether, for instance the rec $^{-}$mutants in $E$. coli (Clark and Margulies, 1965) and the desynaptic types of barley (Riley and Miller, 1966). The "fine" controls can be recognised in various organisms as heterogeneity in recombination values between the same markers, due to differences between the genotypes involved (the "genetic background"). This type of genetic control has been explored in two fungi in particular, namely $\mathcal{N}$ eurospora crassa (Frost, 1961; Lavigne and Frost, 1964; Smith, 1966, 1968; Catcheside, 1968) and Schizophyllum commune (Simchen, 1967; Stamberg, 1968, and personal communication; Simchen and Connolly, 1968) and in both marked variation has been found between different "wild-type" strains. In the latter we have shown that recombination both between $A \alpha$ and $A \beta$ (the two loci that constitute the $A$ incompatibility factor) and between $B \alpha$ and $B \beta$ (the $B$ factor) is subject to genetic control. Different and independent components of the "fine" control system have been shown to be responsible for these two chromosomal regions. In the present paper, we analyse the control of a third chromosomai region which is unlinked to either $A$ or $B$. In addition, the control of recombination in the $A$ chromosome has been subjected to a more detailed analysis by making use of two markers within the $A$ factor (between $A \alpha$ and $A \beta)$ and two outside markers (see fig. 1). Our results suggest that recombination in adjacent regions of the same chromosome may be affected by different components of the "fine" control.

* Present address of both authors: Laboratory of Genetics, The Hebrew University, Jerusalem, Israel. 

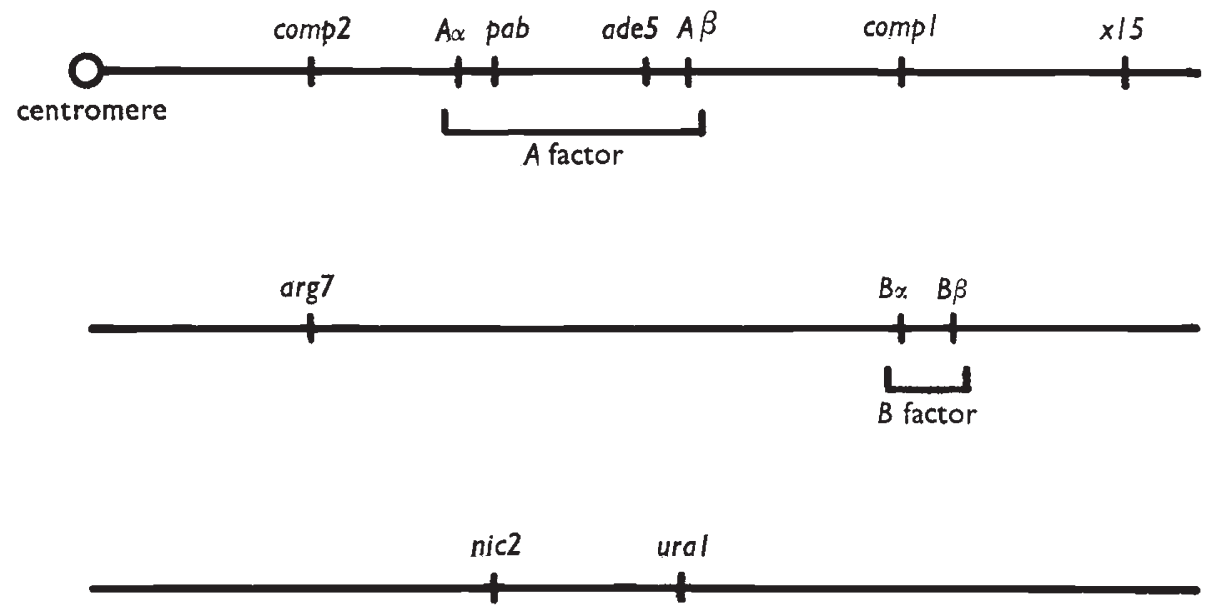

Fig. 1.-The relative order of markers in the three linkage groups of $S$. commune. The symbols of the various markers are:

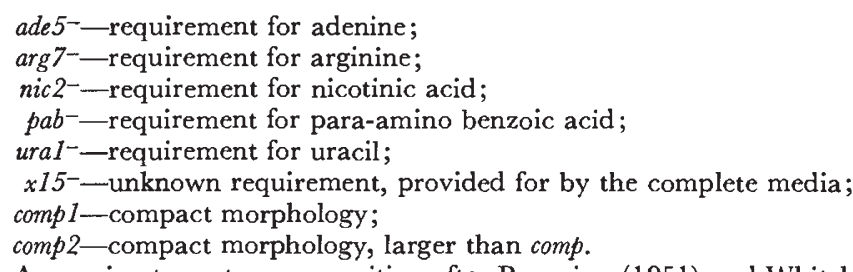

Approximate centromere position after Papazian (1951) and Whitehouse (1957)

\section{Material AND methods}

The strains of $S$. commune used in the present study originated from Professor J. R. Raper's collection in Harvard University (Raper et al., 1960; Koltin et al., 1967) and from the lines selected at Birmingham for high and low recombination frequencies (Simchen, 1967). The details of these strains are given in table 1. The markers used are shown in fig. 1, the actual linkage values not being given because of their variability.

TABLE 1

Details of the main strains used

\begin{tabular}{|c|c|c|c|}
\hline Strain & Mating type & Other markers & Origin \\
\hline 14 & $A 4 B 4$ & & Harvard (fruit body from Ill.) \\
\hline 699 & $A 41 B 41$ & & Harvard (fruit body from Mass.) \\
\hline 991 & $A 97 B 97$ & & Harvard (fruit body from Costa Rica) \\
\hline 1095 & $A 41 B 2$ & nic2- ural- $^{-}$ & Harvard (derived) \\
\hline 1374 & $A 41 B 41$ & $x 15^{-}$ & Harvard (derived) \\
\hline 5219 & $A 43 B 43$ & nic2- uraI- & Birmingham (from E908 $\times 1095$ ) \\
\hline $\mathrm{C} 40$ & $A 43 B 43$ & comp2 $p a b^{-}$ & Birmingham (derived) \\
\hline C41 & $A 43 B 43$ & comp2 $2 a b^{-}$ & Birmingham (derived) \\
\hline E908 & $A 43 B 43$ & & Harvard (fruit body from Ala.) \\
\hline G83 & $A 8 B 7$ & & Birmingham (fruit body from Mass.) \\
\hline G90 & $A 8 B 7$ & & Birmingham (fruit body from Mass.) \\
\hline
\end{tabular}

Note: G83 and G90 were selected for low and high frequencies of recombination within the $A$ factor, respectively (Simchen, 1967). 
The experimental work to be reported here was carried out in three different laboratories, and it is realised that the conditions were not the same throughout. In addition, the two authors started this work independently, using slightly different methods. However, each section of experimental results, which consists of a complete and self-contained set of crosses, was carried out under controlled conditions. Most details concerning culturing media, fruiting, spore-isolation, mating-type tests, etc., can be found in our previous publications (e.g. Simchen, 1967; Stamberg, 1968); strains were screened for biochemical deficiencies on minimal medium (Raper and Miles, 1958) with the appropriate biochemical supplements added as required. Classification of the $p a b^{-}$alleles required an equivalent amount of sulphanilamide to be added to the minimal medium.

Statistical procedures employed were contingency chi-square tests, analyses of variance (of frequencies transformed into angles), linear regression analyses, and correlations. For details see Snedecor (1956).

\section{EXPERIMENTAL RESULTS AND THEIR ANALYSES}

The methodology behind the experiments that are reported in this paper is based on the assumption that one can detect the effects only of loci for which the various genotypes differ in their allelic constitution. Any locus for which only one allele exists in our material will not be recognised, as it cannot show segregation and hence cannot cause heterogeneity in the recombination frequencies observed.

The fruiting of each of the following sets of crosses was carried out at one temperature, and the other environmental conditions were kept as uniform as possible. During the course of the experiments, we learned that in the same genotype different chromosomal regions could react differently to changes in the temperature of meiosis, and that the response of recombination to differences in temperature was also under genetic control; these problems are dealt with elsewhere (Stamberg, 1968, Stamberg and Simchen, 1969).

$$
\text { (i) } A \alpha-A \beta ; B \alpha-B \beta ; \text { nic } 2-u r a 1 \text {. }
$$

As mentioned in the introduction, it has already been established that recombination in the regions $A \alpha-A \beta$ and $B \alpha-B \beta$ is genetically controlled and that distinct components of the "fine" control system are responsible for these two regions (Simchen, 1967; Stamberg, 1968; Simchen and Connolly, 1968). In this section, we shall examine the control of recombination in another, unlinked chromosomal region, the nic2-ural segment which was chosen because it was reported by Raper and Miles (1958) to be unlinked to either $A$ or $B$.

In all the experiments to be reported in this section, fruiting was at $18 \pm 2^{\circ} \mathrm{C}$. The first set of crosses was of G83, G90 and E908 with a common tester designated $1095\left(\right.$ nic2- $2^{-}$ur $\left.^{-}\right)$. The origin and mating types of these strains are given in table 1 , and the results of the crosses are given in table 2. Unfortunately, no recombination in the $B \alpha-B \beta$ region could be observed, as $B 2$ belongs to the nonrecombining group of $B$ factors (Koltin et al., 1967). The results of these crosses, however, suggested that the nic2-ural linkage is under genetic control which is independent of the control of $A \alpha-A \beta$, because G90 differed from E908 with respect to genes affecting the latter 
TABLE 2

Frequencies of recombination of three wild-type strains, all mated to a tester strain 1095. Fruiting at $18^{\circ} \mathrm{C}$.

\begin{tabular}{lccc} 
Strain & Sample size & \multicolumn{2}{c}{ Per cent. recombinants/region } \\
& & $\overbrace{A \alpha-A \beta}$ & nic2-ural \\
G83 & 132 & $2 \cdot 3$ & $3 \cdot 8$ \\
G90 & 150 & $2 \cdot 7$ & $10 \cdot 0$ \\
E908 & 173 & $8 \cdot 1$ & $9 \cdot 3$
\end{tabular}

region only $\left(\chi_{(1)}^{2}=4 \cdot 1\right)$, and at the same time it differed from G83 in recombination in the nic2-ura1 region $\left(\chi_{(1)}^{2}=4.5\right)$. G83 and G90 do not differ in $A \alpha-A \beta$ recombination when mated with 1095, in spite of being selected for low and high recombination, respectively, in this region (Simchen, 1967). It is assumed that 1095 carries a dominant allele for low recombination at the locus in which G83 and G90 differ.

To examine further the gene system controlling the nic2-ura1 region, a single monokaryotic offspring designated 5219 and having the genotype $A 43 B 43$ nic2 $^{-}$ura $1^{-}$was chosen from progeny of the cross E908 $\times 1095$. 5219 was mated to $699(A 41 B 41)$ and gave the following recombination frequencies: $A \alpha-A \beta 7.7$ per cent., $B \alpha-B \beta 0.6$ per cent. and nic2-ura1 11.5 per cent. (sample size 192). Five of the progeny of this cross which were of the $A 41 B 41$ nic2- ura1- genotype were tested for recombination in the three marked regions by mating them to a common tester, $14(A 4 B 4)$.

\section{TABLE 3}

Frequencies of recombination of strain 5219 ( $A 43 B 43$ nic2- ura $1^{-}$) and ten of its offspring from a cross with 699 (genotype of offspring $A 41 B 41$ nic2ura $\left.1^{-}\right)$, all mated to strain $14(A 4 B 4)$. Fruiting at $18^{\circ} \mathrm{C}$.

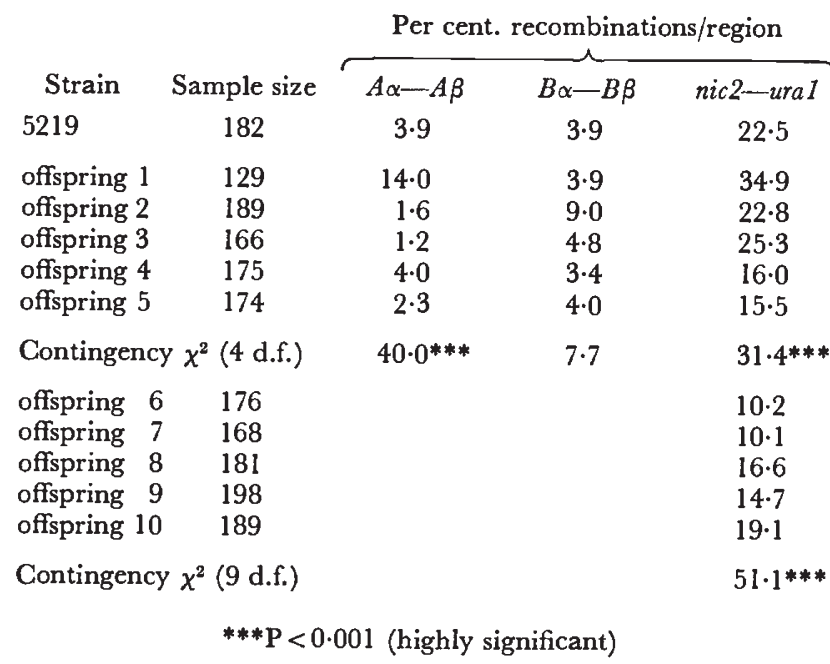

Table 3 demonstrates quite clearly that recombination in nic 2-ura1 is under a separate genetic control from that of $B \alpha-B \beta$, as recombination in the latter region does not vary significantly, in contrast to the former region $(A \alpha-A \beta$ 
seems to change similarly to nic2-ura1, although the correlation is not significantly different from zero; we have, however, established the independence of the controls of these two regions in table 2). Further evidence that recombination in the three chromosomal regions is subject to different control systems is given by the response of the various crosses to a change in the meiotic temperature from $18^{\circ} \mathrm{C}$. to $32^{\circ} \mathrm{C}$. (Stamberg and Simchen, 1969).

To obtain a more detailed knowledge of the control of nic2-ural recombination, five additional progeny of the cross $5219 \times 699$, all of the genotype $A 41 B 41$ nic2- ural $^{-}$, were mated to 14 and tested for recombination in the nic2-ura 1 region only (fruiting at $18^{\circ} \mathrm{C}$.). The recombination frequencies in this region for the ten progeny of the above cross (table 3 ) gave a wide range ( 10 per cent. to 35 per cent.) and could be pooled (by tests of contingency chi-square) only into three or more groups, for instance 6-7, 2-4-5-8-910 and 1-3. This means that at least two loci controlling recombination in the nic2 - ura 1 region were segregating among the progeny of the cross $5219 \times$ 699. From its position relative to this distribution, 5219 is assumed to be +- in respect of these two loci, hence 699 should be -+ .

$$
\text { (ii) } A \alpha-A \beta-x 15 ; B \alpha-B \beta
$$

In the following crosses, frequency of recombination for any particular monokaryon was determined by mating it with a common tester, 1374 $\left(A 41 B 41 \times 15^{-}\right)$and observing the progeny of the dikaryon. We were thus able to test recombination in two adjacent regions, $A \alpha-A \beta$ and $A \beta-x 15$ as well as in the unlinked $B \alpha-B \beta$ segment. Because 1374 , as a recurrent member of each cross, obviously affects recombination in all the dikaryons

TABLE 4

Frequencies of recombination of four " wild-type " strains, all mated to the tester 1374. Fruiting at $18^{\circ} \mathrm{C}$.

\begin{tabular}{|c|c|c|c|c|}
\hline \multirow[b]{2}{*}{ Strain } & \multirow[b]{2}{*}{ Sample size } & \multicolumn{3}{|c|}{ Per cent. recombinants/region } \\
\hline & & $A \alpha-A \beta$ & $A \beta-x 15$ & $B \alpha-B \beta$ \\
\hline G83 & 414 & $2 \cdot 7$ & $7 \cdot 7$ & $1 \cdot 7$ \\
\hline G90 & 413 & $5 \cdot 3$ & $17 \cdot 4$ & $2 \cdot 2$ \\
\hline 14 & 197 & $2 \cdot 0$ & $26 \cdot 9$ & $8 \cdot 1$ \\
\hline E908 & 194 & $13 \cdot 4$ & $26 \cdot 8$ & $3 \cdot 6$ \\
\hline Contin & ncy $\chi^{2}$ (3 d.f.) & $36 \cdot 1 * * *$ & $51 \cdot 8 * * *$ & $19 \cdot 9 * * *$ \\
\hline
\end{tabular}

in which it takes part, the results reported in this section reflect only loci for which 1374 contains the recessive alleles. Loci which are subjected to epistasis by genes in 1374 may also behave as if they were not heterogeneous in the different genotypes.

Fruiting was carried out at $18 \pm 2^{\circ} \mathrm{C}$. At first, four monokaryons, G83, G90, E908 and 14, were tested by examining progeny from their matings with 1374. Frequencies of recombination are given in table 4 . Significant heterogeneity was found in the three regions, namely $A \alpha-A \beta, A \beta-x 15$ and $B \alpha-B \beta$. The results obtained from these four crosses demonstrate that the genotype of the non-common parent influences recombination differently

2 A 2 
in the three regions. The main object in this part of the study was to explore further the relationships between the fine-control components regulating the two adjacent regions. Therefore, the two extreme lines in respect of recombination frequencies in $A \alpha-A \beta$ and $A \beta-x 15$, that is, G83 and E908, were mated, and their progeny grown and tested. This cross gave 1.8 per cent. recombination in the $A \alpha-A \beta$ region; the two other regions could not be examined because both parents were $x 15^{+}$and because they both carried $B \beta 1$. Twenty of the progeny of G83 $\times \mathrm{E} 908$ were taken at random so that their own genotypes, in respect of control of recombination, could be examined. The mating-types of the parents were $A 8 B 7$ (G83) and $A 43 B 43$ (E908), and the twenty monokaryons included five from each of the four

TABLE 5

Frequencies of recombination of iwenty offspring of the cross $G 83$ ( $A 8 B 7) \times$ E908 (A43B43), all mated to the tester $1374\left(A 41 B 41 \times 15^{-}\right)$. Fruiting at $18^{\circ} \mathrm{C}$

\begin{tabular}{ccccc} 
& & \multicolumn{3}{c}{ Per cent. recombinants/region } \\
Offspring & Sample size & $\overbrace{A \alpha-A \beta}$ & $A \beta-x 15$ & $B \alpha-B \beta$ \\
$1(A 8 B 7)$ & 123 & $4 \cdot 1$ & $18 \cdot 7$ & $2 \cdot 4$ \\
$2(A 8 B 7)$ & 131 & $6 \cdot 9$ & $9 \cdot 9$ & $5 \cdot 3$ \\
$3(A 8 B 7)$ & 144 & $8 \cdot 3$ & $20 \cdot 8$ & $0 \cdot 0$ \\
$4(A 8 B 7)$ & 125 & $4 \cdot 0$ & $11 \cdot 2$ & $3 \cdot 2$ \\
$5(A 8 B 7)$ & 132 & $3 \cdot 8$ & $12 \cdot 9$ & $2 \cdot 3$ \\
$6(A 43 B 43)$ & 129 & $12 \cdot 4$ & $14 \cdot 0$ & $3 \cdot 1$ \\
$7(A 43 B 43)$ & 208 & $8 \cdot 2$ & $15 \cdot 4$ & $2 \cdot 4$ \\
$8(A 43 B 43)$ & 206 & $5 \cdot 3$ & $18 \cdot 9$ & $0 \cdot 5$ \\
$9(A 43 B 43)$ & 131 & $2 \cdot 3$ & $9 \cdot 9$ & $3 \cdot 1$ \\
$10(A 43 B 43)$ & 126 & $3 \cdot 2$ & $18 \cdot 3$ & $2 \cdot 4$ \\
$11(A 8 B 43)$ & 133 & $6 \cdot 0$ & $13 \cdot 5$ & $3 \cdot 8$ \\
$12(A 8 B 43)$ & 123 & $5 \cdot 7$ & $9 \cdot 8$ & $2 \cdot 4$ \\
$13(A 8 B 43)$ & 127 & $4 \cdot 7$ & $15 \cdot 8$ & $5 \cdot 5$ \\
$14(A 8 B 43)$ & 135 & $3 \cdot 0$ & $17 \cdot 8$ & $1 \cdot 5$ \\
$15(A 8 B 43)$ & 182 & $1 \cdot 7$ & $7 \cdot 7$ & $2 \cdot 8$ \\
$16(A 43 B 7)$ & 159 & $8 \cdot 8$ & $15 \cdot 1$ & $3 \cdot 1$ \\
$17(A 43 B 7)$ & 151 & $7 \cdot 3$ & $18 \cdot 5$ & $0 \cdot 7$ \\
$18(A 43 B 7)$ & 122 & $4 \cdot 9$ & $11 \cdot 5$ & $0 \cdot 8$ \\
$19(A 43 B 7)$ & 130 & $10 \cdot 0$ & $16 \cdot 2$ & $3 \cdot 1$ \\
$20(A 43 B 7)$ & 149 & $13 \cdot 4$ & $21 \cdot 5$ & $0 \cdot 0$
\end{tabular}

major mating-type groups among the progeny, namely $A 8 B 7, A 8 B 43$, $A 43 B 43, A 43 B 7$. The experiment was designed in this particular way because it was desired to locate genes that affect recombination, and the easiest way was to establish linkage relationships of those genes with the markers which were present in all individual genotypes-the $A$ and $B$ incompatibility factors.

The "recombinational" genotypes of the twenty monokaryons were tested by analysing progeny from a cross of each with the common tester 1374 , as explained above. The results are given in table 5 . In every cross 200-210 spores were isolated but, as in many crosses with 1374 , viability was lower than usual (see sample size in table 5). This reduction in viability affected mainly the $x 15^{-}$class; that is to say, when viability was low, there were usually fewer auxotrophs than prototrophs. None of the other markers showed a disturbed segregation as a result of a differential viability of its own 
— the $A \alpha$ and $A \beta$ loci reflected of course their linkage to $x 15$. When unequal viability exists at one locus only, the recombination frequencies can be calculated in the usual way (recombinants/total) without any statistical complications (Bailey, 1961, pp. 50-51).
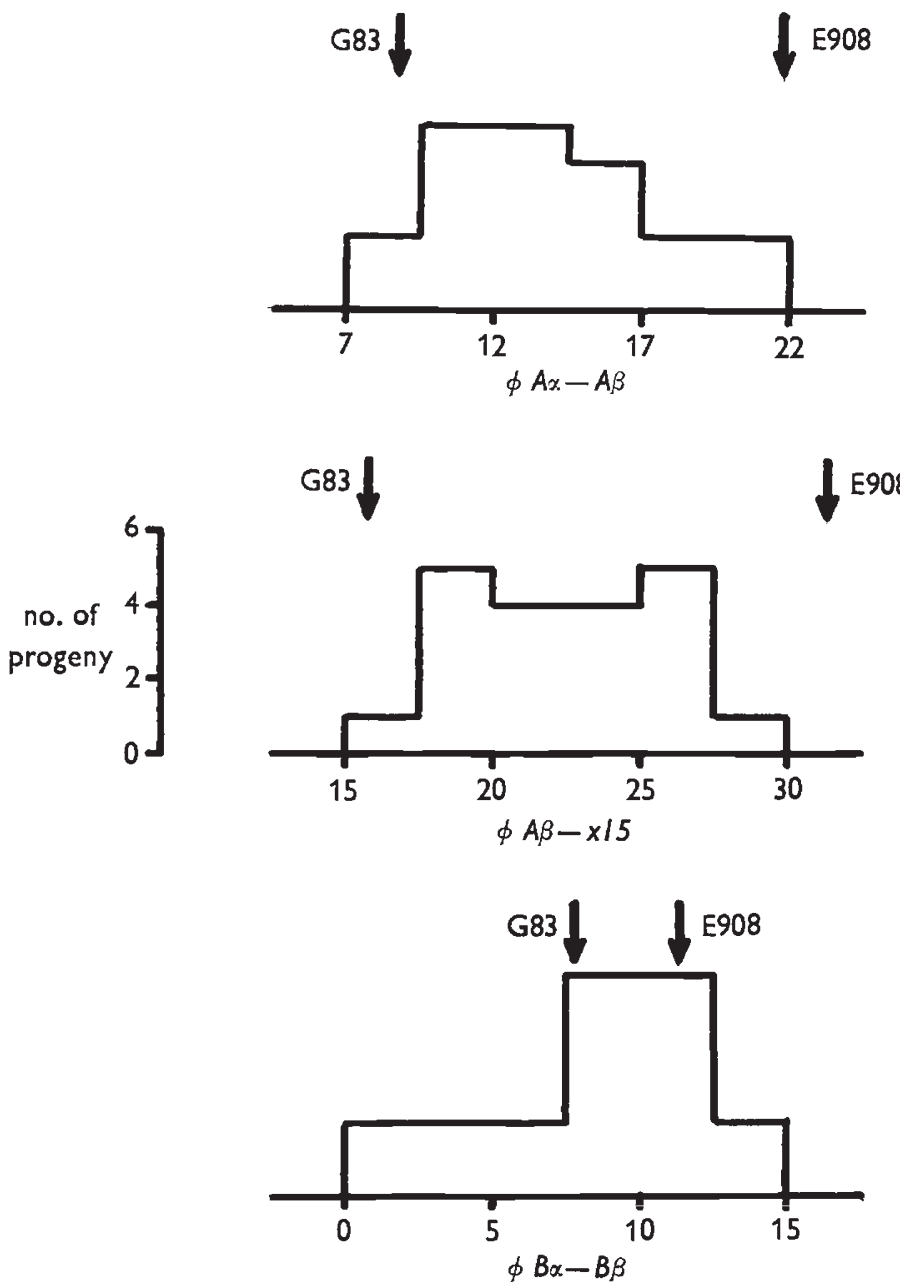

FIG. 2.-Distributions of recombination values (percentages transformed into angles- $\phi$ ) of the twenty offspring of the cross G83 $\times$ E908. Parental values are marked by arrows.

Before the data were analysed by the common statistical procedures, which assume that the accuracy of different observations is more or less the same, they were transformed into angles $\phi$ by $p=\sin ^{2} \phi$, where $p$ is the observed frequency of recombination. If this had not been done, the variance of $p$ would have been $p(1-p) / n$, i.e. dependent on $p$, while the variance of $\phi$ when measured in degrees is $820.7 / n$, i.e. it is independent of either $p$ or $\phi$. A harmonic mean $\bar{n}$ was used to obtain a joint theoretical error variance, by which the sum of squares of various sources of variation could be divided in order to give a chi-square. 
The 20 monokaryons provided us with an analysis of variance with 19 degrees of freedom (d.f.), 3 of which can be allocated to comparisons between the four mating-type groups, whereas the remaining 16 represented the variation within these groups. Of the above-mentioned 3 d.f. for a comparison between the mating-type groups, two provide information about linkage: one d.f. for the comparison $A 8$ vs. $A 43$, which tests for linkage of " recombinational "genes to the $A$ factor, the other for $B 7$ vs. $B 43$, testing for linkage to the $B$ factor. The results of these comparisons for all three chromosomal regions showed that there was no case for claiming linkage to $B$, and only "borderline" evidence for linkage to $A$ of genes which affected recombination in $A \alpha-A \beta$ (probability of 5 to 10 per cent.).

The distributions of recombination values are plotted in fig. 2 . In respect of the $A \alpha-A \beta$ region, the parental values are at the extreme ends of

\section{TABLE 6}

Correlation analysis of the data given in table 5 (transformed into angles) between any two of the three chromosomal regions. Given are the correlation coefficients $\mathrm{r}$ with their probabilities ( $\mathrm{P}$ for 18 d.f.), and the chi-squares (also with 18 d.f.) testing the remainder of the variation in $y$ against the theoretical error $(=5 \cdot 875)$.

\begin{tabular}{|c|c|c|c|c|}
\hline & \multicolumn{3}{|c|}{ Region $x$} \\
\hline & & $A \alpha-A \beta$ & $A \beta-x 15$ & $B \alpha-B \beta$ \\
\hline & $A \alpha-A \beta$ & & $\begin{array}{l}r=0.4354 \\
\mathrm{P}=0.10-0.05 \\
\chi^{2}=37.93 \\
\mathrm{P}=0.01-0.001\end{array}$ & $\begin{array}{l}r=-0.2403 \\
\mathrm{P}>0.10 \\
\chi^{2}=44.09 \\
\mathrm{P}<0.001\end{array}$ \\
\hline egion $y<$ & $A \beta-x 15$ & $\begin{array}{l}r=0.4354 \\
\mathrm{P}=0 \cdot 10-0.05 \\
\chi^{2}=28.52 \\
\mathrm{P}=0 \cdot 10-0.05\end{array}$ & & $\begin{array}{l}r=-0.5746 \\
\mathrm{P}=0.01-0.001 \\
\chi^{2}=23.57 \\
\mathrm{P}>0.10\end{array}$ \\
\hline & $B \alpha-B \beta$ & $\begin{array}{l}r=-0.2403 \\
\mathrm{P}>0.10 \\
\chi^{2}=31.45 \\
\mathrm{P}=0.05-0.02\end{array}$ & $\begin{array}{l}r=-0.5746 \\
P=0.01-0.001 \\
\chi^{2}=22.36 \\
P>0.10\end{array}$ & \\
\hline
\end{tabular}

the distribution, and it is therefore assumed that at least two loci control recombination in this region (the parents being ++ and -- ). A similar situation exists for the $A \beta-x 15$ region, but here the distribution is bimodal, which means that one relatively large effect on recombination is segregating. From the relative position of the parental values and the two modes of the distribution, it is suggested that at least one additional locus with a small effect is also segregating. The third distribution, that concerning the $B \alpha-B \beta$ region, is again unimodal, though the parental values differ only slightly (and non-significantly- $\chi_{(1)}^{2}=2 \cdot 16$ ). There is, however, significant variation among the progeny, which means that at least two loci are segregating among them, the parents being +- and -+ in respect of these loci.

In order to examine the joint variation in recombination frequencies in different regions, correlation coefficients were calculated for every combination of two of the three segments (table 6). The remaining variation in any one region was tested against a theoretical error, to reveal significant, uncorrelated, effects on recombination. 
On the basis of these results the following model for the control of recombination in the three regions is proposed (fig. 3). This model is tentative and is given as an example of the kind of interactions that could explain the data.

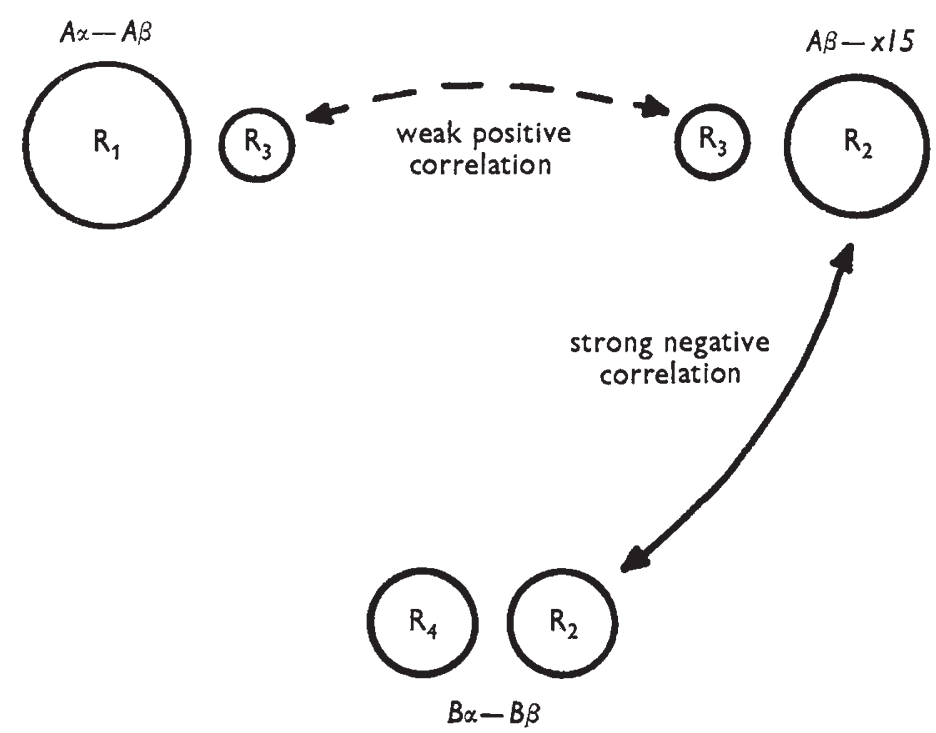

Fig. 3.-A proposed model for control of recombination revealed by the data of table 5 (see text for details).

$\mathrm{R}_{1}$-consists of at least two loci of equal effect. It is responsible for the major part of the variation in recombination values observed in $A \alpha-A \beta$. The two parents, E908 and G83, carry the extreme combinations of the two loci, i.e. ++ and -- respectively.

$\mathrm{R}_{2}$-one gene with a large effect on $A \beta-x 15$ (causing bimodality of the distribution of progeny) and affecting $B \alpha-B \beta$ in the opposite way, probably to a lesser extent.

$\mathrm{R}_{3}$ - the almost significant correlation between the $A \alpha-A \beta$ and $A \beta-x 15$ regions may result from at least one locus affecting both. There is also the indication for possible linkage of genes affecting the $A \alpha-A \beta$ region to the $A$ factor, which may be due to this system or to an element of $\mathrm{R}_{\mathrm{t}}$. $\mathrm{R}_{4}$--probably (at least) one additional locus affecting $B \alpha-B \beta$ to about the same extent as $R_{2}$. This is supported by the distribution of the progenies in relation to their parents, not by the correlation analysis. With respect to recombination in $B$, the two parents are E908 $\mathrm{R}_{2}-\mathrm{R}_{4}+$ and G83 $\mathrm{R}_{2}{ }^{+} \mathrm{R}_{4}{ }^{-}$(but the effect of $\mathrm{R}_{2}$ on $A \beta-x 15$ is of course the opposite).

$$
\text { (iii) } A \alpha-p a b-a d e 5-A \beta ; B \alpha-B \beta
$$

Two nutritional markers - $p a b$ and ade 5-are located between the $A \alpha$ and $A \beta$ loci (fig. 1). These four loci were utilised in a number of crosses designed to answer the following questions:

(1) Does the gene system controlling recombination between $A \alpha$ and $A \beta$ affect the whole region evenly, or does it mainly affect one or two of the three sub-regions $(A \alpha-p a b, p a b-a d e 5$, ade $5-A \beta)$ ? 
(2) Is there evidence for interference of crossing-over within the $A$ factor, and if so, is the intensity of interference perhaps an agent by which the genotype affects the frequency of recombination?

A tester strain of the genotype $A \alpha 1 p a b^{-} a d e 5^{-} A \beta 6 B 41$ was used throughout the experiments reported in this section. Strain 14 and five offspring of the cross $14 \times 699$, all of mating-type $A 4 B 4$, were mated to the tester strain, and samples of their progeny grown and analysed in order to determine the frequency of recombination in the various chromosomal regions. The six dikaryons that resulted from these matings were each fruited at two temperatures $-23 \pm 1^{\circ} \mathrm{C}$. and $32 \pm 1^{\circ} \mathrm{C}$. No variation in frequencies of recombination could be observed at the lower temperature in any of the three sub-regions of the $A$ factor, giving pooled values of 0.4 per cent. for recombination frequency in $A \alpha-p a b, 3.5$ per cent. in $p a b-a d e 5$ and 1.6 per cent. in ade $5-A \beta$

TABLE 7

Frequencies of recombination of strain $14(A 4 B 4)$ and five of its offspring from a cross with 699 (genotype of offspring $A 4 B 4)$, all mated to a tester strain of the genotype $A \alpha 1$ pab-ade5- $A \beta 6 B 41$. Fruiting at $32^{\circ} \mathrm{C}$.

\begin{tabular}{|c|c|c|c|c|c|}
\hline Strain & Sample size & $A \alpha-p a b$ & $p a b-a d e 5$ & ade $5-A \beta$ & $B \alpha-B \beta$ \\
\hline 14 & 379 & $2 \cdot 1$ & $10 \cdot 5$ & $2 \cdot 6$ & $2 \cdot 4$ \\
\hline Offspring 1 & 120 & $0 \cdot 8$ & $9 \cdot 2$ & $6 \cdot 7$ & $9 \cdot 2$ \\
\hline Offspring 2 & 184 & $2 \cdot 7$ & $28 \cdot 2$ & $4 \cdot 4$ & $4 \cdot 4$ \\
\hline Offspring 3 & 216 & $5 \cdot 6$ & $15 \cdot 3$ & $3 \cdot 2$ & $5 \cdot 6$ \\
\hline Offspring 4 & 169 & $3 \cdot 0$ & $14 \cdot 2$ & $3 \cdot 0$ & $1 \cdot 8$ \\
\hline Offspring 5 & 187 & $4 \cdot 3$ & $8 \cdot 6$ & $4 \cdot 8$ & $6 \cdot 4$ \\
\hline $\begin{array}{l}\text { Contingency } \\
\text { Probability }\end{array}$ & (5 d.f.) & $\begin{array}{c}14 \cdot 7 \\
0 \cdot 02-0 \cdot 01\end{array}$ & $\begin{array}{l}42 \cdot 0 \\
<0 \cdot 001\end{array}$ & $\begin{array}{c}5 \cdot 0 \\
0 \cdot 50-0 \cdot 30\end{array}$ & $\begin{array}{c}15 \cdot 5 \\
0.01-0.001\end{array}$ \\
\hline
\end{tabular}

(based on 1471 progeny). There was, however, significant variation in recombination frequencies in the $B \alpha-B \beta$ region $\left(\chi_{(5)}^{2}=12.58, \mathrm{P}=\right.$ $0.05-0.01$ ) with values from 2.8 per cent. to 10.5 per cent. By contrast, the higher meiotic temperature $\left(32^{\circ} \mathrm{C}\right.$.) induced pronounced heterogeneity in frequencies of recombination in two of the sub-regions of $A$ as well as in the $B$ factor (table 7 ). We shall not discuss further the effect of the change in temperature on recombination in the various genotypes as this is done elsewhere (Stamberg and Simchen, 1969). Table 7 shows quite clearly that genotypic variation in frequencies of recombination in $A \alpha-p a b$ and $p a b-a d e 5$ does not affect the ade $5-A \beta$ sub-region. Moreover, the two former subregions do not appear to be controlled by exactly the same gene systems: offspring $l$ is evidently the source of heterogeneity in recombination values in $A \alpha-p a b\left(\chi_{(4)}^{2}\right.$ for the other five genotypes is 5.85, which is not significant), while offspring 2 is the source of heterogeneity in the pab-ade 5 sub-regions $\left(\chi_{(4)}^{2}\right.$ for the other genotypes is $6 \cdot 76$, again not significant).

It appears that in the present set of experiments at least one locus affects the $A \alpha-p a b$ sub-region and at least one other locus affects the $p a b$-ade 5 sub-region, whereas there is no evidence for genetic control of recombination between ade5 and $A \beta$. Strain 14 probably has the allele that increases recombination in the locus affecting $A \alpha-p a b$ and a "decreasing " allele at the other locus (affecting pab-ade5), while 699 probably possesses the alternative alleles at those two loci. 
As for the question of interference, there were no discrepancies between observed and expected numbers of double crossovers in adjacent regions. It has been concluded, therefore, that interference could not be the agent by which the genetic control system affects recombination frequencies.

$$
\text { (iv) comp2- } A \alpha-p a b-A \beta ; B \alpha-B \beta
$$

Because the preceding experiments showed that the fine-control components regulating recombination within the $A$ factor affected those parts of the segment near $A \alpha$, the region adjacent to $A \alpha$ but outside the $A$ factor was

TABLE 8

Frequencies of recombination obtained from five different crosses between $\mathrm{C} 40$ and $\mathrm{C} 41$, both $A 43 B 43$ comp2 pab-, and three wild-type strains, 699, 14 and 991 . Fruiting at $32^{\circ} \mathrm{C}$.

\begin{tabular}{|c|c|c|c|c|c|}
\hline \multirow[b]{2}{*}{ Cross } & \multirow[b]{2}{*}{ Sample size } & \multicolumn{4}{|c|}{ Per cent. recombinants/region } \\
\hline & & $\operatorname{comp} 2-A \alpha$ & $A \alpha-p a b$ & $p a b-A \beta$ & $B \alpha-B \beta$ \\
\hline 1. $\mathrm{C} 40 \times 699$ & 117 & $19 \cdot 7$ & $0 \cdot 9$ & $18 \cdot 0$ & $0 \cdot 0$ \\
\hline 2. $\mathrm{C} 41 \times 699$ & 168 & $31 \cdot 6$ & $1 \cdot 2$ & $17 \cdot 3$ & $2 \cdot 4$ \\
\hline 3. $\mathrm{C} 40 \times 14$ & 190 & $19 \cdot 5$ & $2 \cdot 1$ & $13 \cdot 2$ & $3 \cdot 2$ \\
\hline 4. $\mathrm{C} 41 \times 14$ & 201 & $21 \cdot 9$ & $7 \cdot 0$ & $11 \cdot 0$ & $3 \cdot 5$ \\
\hline 5. C41 $\times 991$ & 126 & $21 \cdot 4$ & $6 \cdot 3$ & $19 \cdot 1$ & $5 \cdot 6$ \\
\hline \multirow{2}{*}{\multicolumn{2}{|c|}{$\begin{array}{l}\text { Contingency } \chi^{2} \text { ( } 4 \text { d.f.) } \\
\text { Probability }\end{array}$}} & $9 \cdot 4$ & $15 \cdot 8$ & 6.9 & $6 \cdot 9$ \\
\hline & & $0 \cdot 10-0 \cdot 05$ & $0.01-0.001$ & $0 \cdot 20-0 \cdot 10$ & $0 \cdot 20-0 \cdot 10$ \\
\hline
\end{tabular}

next studied (see fig. 1). The aim was to determine whether the effect of the genes controlling recombination within the $A$ factor extended proximally beyond it.

C40 and C41, two sibling strains of the genotype $A 43 B 43$ comp2 pab ${ }^{-}$, were obtained and mated with three wild-type strains, 699, 14 and 991 (Table 8).

The genotypic differences between the various crosses affect recombination only in the $A \alpha-p a b$ sub-region, i.e. there is evidence for genes that control recombination in this segment and not in the two adjacent segments, comp $2-A \alpha$ and $p a b-A \beta$. Thus the effect of genes that control recombination in the $A \alpha-p a b$ region does not extend beyond this region in either direction.

\section{Discussion}

The conclusions drawn from the experiments reported in the preceding sections are as follows:

(1) Recombinational events in unlinked chromosomal segments are controlled by different components of the fine control (e.g. $A \alpha-A \beta, B \alpha-B \beta$ and nic2-ura1).

(2) Adjacent regions may also be controlled by different components (e.g. $A \alpha-p a b, p a b-a d e 5)$; in other words, the loci that control recombination are highly region-specific.

(3) There are, however, some loci that control recombination in more than one region, either coordinately or in a contrasting manner (i.e. the same allele increases recombination in one region and decreases it in another, unlinked region, for instance $A \beta-x 15$ and $B \alpha-B \beta$.) 
Our present understanding of the genetic control of recombination in Schizophyllum, though still far from complete, is that the effects of genes controlling the frequency of recombination are localised in chromosomal regions that are rather limited in length. This would require a large number of genes to control recombination, but the results reported here show us that some genes can take part in the control of several segments, thus making the total number of controlling genes not unreasonably high. The exact nature of the relationship between the controlled segments and their controlling genes is unknown at present, but because the regulation of recombination is region-specific, some means of recognition must be involved. The implications of these findings have been discussed in more detail elsewhere (Simchen and Stamberg, 1969).

\section{Summary}

1. Genetic control of recombination in adjacent and non-adjacent chromosomal regions has been studied by making use of several strains of Schizophyllum commune in a series of crossing programmes.

2. It has been shown that recombination in unlinked chromosomal segments is regulated by different components of the control system.

3. Adjacent regions are also controlled by different components, i.e. the effect of certain loci on recombination seems to be localised in short segments.

4. There is evidence that at least some loci control recombination in more than one region.

5. It is suggested that each of the numerous controlling genes must have some specific relationship to the short chromosomal segment in which it affects recombination.

Acknowledgments.-We would like to thank Miss Judy Hay and Miss Rachel Ronen for technical assistance and our colleagues who read the manuscript and made numerous valuable suggestions: R. Falk, J. L. Jinks, Y. Koltin, J. R. Raper, E. C. R. Reeve and H. L. K. Whitehouse.

This work was partly financed by the Agricultural Research Council of Great Britain and by a U.S. Public Health Service Predoctoral fellowship from the National Institute of General Medical Sciences to the junior author.

\section{REFERENCES}

BAILEY, N. 1961. Introduction to the Mathematical Theory of Genetic Linkage. Clarendon Press, Oxford.

CATcheside, D. G. 1968. The control of genetic recombination in Neurospora crassa. In Replication and Recombination of Genetic Material (ed. W. J. Peacock and R. D. Brock), Canberra (Australia).

CLARK, A. J., AND MARGULIES, A. D. 1965. Isolation and characterization of recombination deficient mutants of Escherichia coli K12. Proc. Natl. Acad. Sci. U.S., 53, 451-459.

FROST, L. C. 1961. Heterogeneity in recombination frequencies in Neurospora crassa. Genet. Res., 2, 43-62.

KOLTIN, Y., RAPER, J. R., AND SIMCHEN. G. 1967. The genetic structure of the incompatibility factors of Schizophyllum commune: The B factor. Proc. Natl. Acad. Sci. U.S., 57, 55-62.

LAVIGNE, s., AND FROST, L. c. 1964. Recombination frequency and wildtype ancestry in linkage group I of Neurospora crassa. Genet. Res., 5, 366-378.

Papazian, H. P. 1951. The incompatibility factors and a related gene in Schizophyllum commune. Genetics, 36, 441-459.

RAPER, J. R., BAXTER, M. G., AND EILINGBoE, A. H. 1960. The genetic Structure of the incompatibility factors of Schizophyllum commune: The A factor. Proc. Natl. Acad. Sci. U.S., 46, 833-842. 
RAPER, J. R., AND MILES, P. G. 1958. The genetics of Schizophyllum commune. Genetics, 43, 530-546.

RILEY, R., AND MILLER, T. E. 1966. The differential sensitivity of desynaptic and normal genotypes of barley to X-rays. Mutation Res., 3, 355-359.

SIMCHEN, G. 1967. Genetic control of recombination and the incompatibility system in Schizophyllum commune. Genet. Res., 9, 195-210.

SIMGHEN, G., AND CONNOLLY, v. 1968. Changes in recombination frequency following inbreeding in Schizophyllum. Genetics, 58, 319-326.

simchen, G., AND STAMBERG, J. 1969. Fine and coarse controls of genetic recombination. Nature, 222, 329-332.

SMr'rH, B. R. 1966. Genetic controls of recombination. I. The recombination-2 gene of Neurospora crassa. Heredity, 21, 481-498.

sмiтн, в. R. 1968. A genetic control of recombination in Neurospora crassa. Heredity, 23, 162-163 (Abst.).

SNEDECOR, G. W. 1956. Statistical Methods (5th edition). Iowa State Univ. Press, Ames (Iowa).

Stamberg, J. 1968. Two independent gene systems controlling recombination in Schizophyllum commune. Molec. Gen. Genet., 102, 221-228.

STAMBERG, J., AND simchen, G. 1969. Specific effects of temperature on recombination in Schizophyllum commune. Heredity (in press).

Whitehouse, H. L. K. 1967. Mapping chromosome centromeres from tetratype frequencies. J. Genet., 55, 348-360. 